\title{
Las nuevas pautas para el acceso a la información
}

\author{
Ariel Alejandro Rodríguez García *
}

Artículo recibido:

7 de noviembre de 2012.

Artículo aceptado:

14 de mayo de 2015.

\section{Resumen}

El título del estudio sugiere que con el desarrollo de la Web 2.0 y las tecnologías de la información han surgido nuevos modelos de producción, distribución y consumo de información. De ahí que el propósito del trabajo sea examinar los cambios que se han suscitado en el acceso a la información tanto para usuarios como para profesionales de la información. La tesis del estudio centra su atención en los señalamientos de Kuhn respecto a la prioridad de los paradigmas, la cual refiere que los nuevos profesionales adoptarán las prácticas emergentes sin cuestionar las causas que le dieron origen. La comprobación se hace por medio de los postulados de Rifkin sobre la economía-red y los vertidos

* Instituto de Investigaciones Bibliotecológicas y de la Información de la UNAM, México. ariel@iibi.unam.mx

INVESTIGACIÓN BIBLIOTECOLÓGICA, Vol.30, Núm.69, mayo/agosto, 2016, México, ISSN: 0187-358X. pp. 121-141 
por Vargas Llosa con relación a la metamorfosis de la cultura escrita para la red. Los resultados que se presentan en el estudio se derivan del proyecto PAPIIT IT 400312 Biblioteca Digital en Bibliotecología y Estudios de la Información.

Palabras claves: Acceso a la información; Prosumidores; Web 2.0.

\section{Abstract}

\section{New directions in information access}

Ariel Alejandro Rodríguez-García

With the development of Web 2.0 and information technologies, new models of production, distribution and consumption of information have emerged. This study examines the changes undergone in information access for both general users and information professionals. The study's approach is informed by Kuhn's contention regarding the priority of paradigms, in which neophyte professionals will adopt emerging practices without questioning their causes or origins. This hypothesis is tested by way of Rifkin's postulates on the network economy and those proffered by Vargas Llosa regarding the metamorphosis of written culture for the network. The results presented are derived from the project PAPIIT IT 312400 Digital Library in library science and information studies.

Keywords: Access to Information; Podusage; Web 2.0 .

\section{INTRODUCCIÓN}

$\mathrm{E}^{\mathrm{l}}$ 1 acceso a la información ha sido analizado desde distintas disciplinas, entre las que se encuentran la bibliotecología y estudios de la información. De los asuntos revisados se ha discurrido sobre el copyright, los códigos abiertos, la privacidad y seguridad de los datos, así como el dominio que está ejerciendo la información digital en las nuevas prácticas para producirlos, distribuirlos y consumirlos. 
El interés de prestar atención a lo que está sucediendo con el acceso a la información tiene como finalidad interpretar cómo la biblioteca digital, con su colección organizada y con servicios asociados, debe asumir que los modelos librarios no son los más apropiados para explicar lo que está sucediendo en el entorno digital, sino que están emergiendo otros que no se asemejan a los vigentes.

El reciente enfoque sobre la naturaleza de la biblioteca digital está interesándose en el uso de las tecnologías de la información y las aplicaciones tecnológicas emergentes, al tiempo que ha descuidado los fundamentos de aquélla, situación que está llevando a un empirismo. Lo anterior confirma la hipótesis de Shera, quien sostiene que los nuevos procedimientos de miniaturización de alta reducción parecen alcanzar cualquier límite, así como aquélla otra planteada por Rifkin que subraya que así como la imprenta alteró la conciencia humana durante los últimos siglos, la tecnología de la información probablemente tendrá un efecto similar sobre las conciencias en los años por venir.

El planteamiento sobre las nuevas pautas para el acceso a la información surge a partir del modelo de biblioteca digital sustentada en el arquetipo de la Web 2.0, la cual aprovecha al máximo las aplicaciones tecnológicas actuales que están siendo empleadas para producir y consumir la información digital en sistemas de información abiertos. En este sentido, toma relevancia el supuesto que señala que el acceso a la información digital en un contexto abierto permitirá que tanto los usuarios tradicionales como aquellos que están identificándose como prosumidores podrán producir, consumir y compartir información a través de redes, las cuales dispondrán de diversos rudimentos que les enseñarán y guiarán para lograr su convergencia cultural en entornos digitales.

Agradecemos a los coordinadores del Proyecto PAPIIT IT400312 Biblioteca Digital en Bibliotecología y Estudios de la Información el financiamiento para entregar los presentes resultados de investigación.

\section{TRES PERSPECTIVAS SOBRE EL ACCESO A LA INFORMACIÓN}

La economía sustentada en el acceso, el vertiginoso desarrollo de la tecnología de la información y comunicación que modifica los hábitos en el consumo de la información y la resignificación de la naturaleza de las entidades en la organización de la información son muestra de los diversos eventos que en los últimos años han propiciado el cambio drástico en el acceso a la información. 
La economía en red, como la ha denominado Rifkin, ${ }^{1}$ tiene como rasgo esencial la conectividad. A diferencia de la establecida por la era industrial, la cual necesitaba de un lugar geográfico para llevar a cabo las negociaciones y transacciones, esta economía se desenvuelve a través del intercambio de datos en el ciberespacio. Establece nuevos modelos de organización en la vida económica, se gobierna por redes más que mercados, los vendedores y compradores son sustituidos por proveedores y usuarios y prácticamente todos los productos adquieren el rasgo de acceso.

En el entorno de las tecnologías de la información, del mismo modo que en la economía, los cambios que se perciben están presentes en los individuos (usuarios de la información), quienes dejan de ser solamente consumidores pasivos y pasan a ser participantes activos en, por ejemplo, la producción de contenidos digitales.

A los nuevos usuarios de la información digital se les está conociendo como prosumidores de información. Según Burns, ${ }^{2}$ un prosumidor participa activamente en la producción de contenidos a través de los contenidos comunitarios generados al interior de las redes sociales. Aún pueden estar presentes usuarios tradicionales o híbridos; en términos comunes, éstos siguen siendo consumidores pasivos debido a que no han llegado al extremo de producir y consumir la información al mismo tiempo.

A propósito de esto último, traeremos a la reflexión algunas preguntas expresadas por Vargas Llosa con relación a la producción del libro: ¿sobrevivirán los libros de papel o acabarán con ellos los libros electrónicos? ¿Los lectores del futuro serán sólo de tabletas digitales? De forma angustiada, Vargas Llosa ${ }^{3}$ señala que el arribo del libro electrónico es inminente, al extremo de que es imposible evitar una época en que los lectores de libros de pantalla sean la gran mayoría y los de papel queden reducidos a ínfimas minorías o incluso desaparecerán, al igual que librerías, bibliotecas, editoriales, agentes literarios, correctores, distribuidores, y sólo quedará la nostalgia. Finaliza diciendo que cuando los escritores escriban literatura virtual no lo harán como se ha venido haciendo, en pos de la materialización de sus escritos en un objeto concreto, táctil y durable que es (nos parece) el libro.

En lo concerniente a la resignificación en la naturaleza de las entidades, diremos que desde la perspectiva de la organización de la información la justificación vertida por Svenonius ${ }^{4}$ sobre el lenguaje del documento invita a fijarse en que los atributos descriptivos son los más indicados para permitir el 
acceso a la información, puesto que hay otros que responderán al lenguaje de la obra y que son los que permiten el acceso al contenido. Un buen lenguaje del documento, nos dice Svenonius, ${ }^{5}$ ayuda en su localización, asiste en su identificación, favorece la selección y adquisición y permite la navegación entre el mismo documento.

Sin duda estas tres perspectivas sobre el acceso a la información son propicias para plantear el argumento central de este trabajo. Con el desarrollo de la información digital y las características que hacen distintivos a los recursos de información digital es posible establecer nuevas pautas en el acceso a la información. De ser así, ¿cuál es el rol que está jugando la resignificación del lenguaje del documento? ¿De qué forma los nuevos usuarios asumen que la información digital, para que esté activa en cualquier sistema de información, requiere de su activa participación en el etiquetado de los recursos de información?

\section{Atributos Del acceso a la información}

Es de interés para este trabajo ahondar sobre los atributos del acceso desde dos temas. El primero se dirige hacia lo que Svenonius señala sobre los cambios en el lenguaje del documento a raíz de los nuevos atributos de los recursos de información y las actualizaciones en el modelo de catalogación; el segundo se remite al tema de la recuperación de la información vista desde lo que Burns refiere sobre el prosumidor de información y la forma en que son creadas las nuevas pautas tanto para la producción como para el consumo de la información digital.

\section{El lenguaje del documento: atributos para el acceso}

Es cierto que el lenguaje del documento ha cambiado desde la aparición de los documentos electrónicos y luego con el documento digital. Esto ha sido a tal grado que el modelo de catalogación que se tenía conceptuado de acuerdo con las Reglas de Catalogación Angloamericanas, segunda edición (RCAA2), tuvo que modificarse porque era difícil adaptar el Principio Cardinal ${ }^{6}$ a los atributos de esos nuevos documentos. De ahí que en la catalogación, desde la década de los noventa del siglo pasado, considere a las tecnologías de la

5 Ibid., 108.

6 El Principio Cardinal fue concebido para gobernar el proceso de transcripción, de despliegue y las opciones de acceso a los objetos bibliográficos que como característica común tienen un formato físico. 
información como el mediador entre la manera de describir al documento electrónico y digital y la forma de acceder a la información y contenidos.

De distintas formas se ha señalado que el Principio Cardinal es una de las grandes tradiciones de la catalogación, pero en la actualidad su rigidez impide que los nuevos documentos sean descritos con amplitud. Por esta razón fue necesario actualizar las diversas normativas para la descripción bibliográfica y crear el modelo de los Requerimientos Funcionales ${ }^{7}$ como el principal artífice del cambio.

Conviene subrayar que mientras se desarrollaba el modelo conceptual hubo quienes predecían que, con la llegada del nuevo milenio, la organización de la información estaría en graves problemas si no se atendía la aparición del documento digital, a tal grado que podría comprometerse el futuro de los sistemas de recuperación de información. ${ }^{8}$

El modelo introducido por la IFLA reconoce que toda aquella entidad de información que se encuentra en el universo bibliográfico puede ser descrita y representada sin importar su naturaleza. Con este argumento se está frente a un nuevo conjunto de supuestos y uno de los principales es que todo atributo de cualquier entidad, por ejemplo la digital, permite el acceso y la recuperación de datos sin importar su naturaleza, sea ésta intrínseca o extrínseca.

Es evidente que la información digital ha sumado al universo bibliográfico nuevas formas y formatos para almacenar información, se ha puesto en la mesa de discusión la manera de comprender y relacionarse con la información que posee un objeto intangible, se han establecido las formas básicas para aquellos que organizan información con el fin de que realicen su trabajo a partir de la unidad básica, el conjunto de entidades o los recursos de información digital. Es decir, por medio de sus particularidades (atributos) identificadas como elementos lógicos ${ }^{9}$ para el acceso e identificación, así como para la recuperación en un sistema de información.

En lo que respecta al modelo de los Requerimientos Funcionales, nos parece importante referir que después de tres lustros de su aparición ha comenzado a emplearse como marco teórico en la estructura y contenido de los Lineamientos para la Descripción y Acceso a los Recursos (RDA por sus siglas en inglés), sustitutos de las RCAA2.

7 IFLA Study Group on the Functional Requirements for Bibliographic Records. El grupo de trabajo FRBR tuvo como objetivos proponer un marco estructurado y claramente definido para relacionar los datos consignados en los registros bibliográficos con las necesidades de los usuarios y recomendar un nivel básico de funcionalidad de los registros creados por las agencias bibliográficas nacionales.

8 Ariel Alejandro Rodríguez García, Las nuevas entidades de información analizadas desde la perspectiva de la organización de la información, 11.

9 Linda Schamber, "What is a document? Rethinking the concept in uneasy time", 669. 
En palabras de Oliver ${ }^{10}$ los nuevos lineamientos son un estándar diseñado para poner atención en los usuarios y las tareas que realizan cuando emplean los descubridores de recursos, por lo que este modelo constituye una nueva alternativa para reflexionar acerca de los datos bibliográficos y de autoridad.

A propósito de los lineamientos, Oliver ${ }^{11}$ refiere que fueron creados para que la administración de los datos pueda usar tanto las tecnologías actuales como aquellas que estructuran las bases de datos que van surgiendo con el fin de atender a las tecnologías futuras. En este sentido, los lineamientos para la descripción y acceso proponen que las particularidades de los recursos puedan abarcar tanto a los tradicionales como no tradicionales, a los recursos análogos como digitales, así como aquellos que están dentro o fuera del entorno bibliotecario.

Lo que nos gustaría particularizar sobre los lineamientos y su relación con los atributos para el acceso a la información es que los lineamientos han sido propuestos para que distintas comunidades los empleen como un estándar de descripción; para que se entienda que su flexibilidad y extensión permiten a las distintas estructuras de metadatos contar con los elementos necesarios para habilitar los atributos de las entidades con el fin de almacenar, transmitir e intercambiar los datos empleando diversos esquemas de codificación, los cuales traerán beneficios a los usuarios finales.

El tema que no se puede pasar por alto y que tal vez, desde la perspectiva de la catalogación, nos permita comprender con mayor precisión los atributos del acceso, son los metadatos. Para la organización de la información los metadatos juegan un papel importante, ya que desde la llegada de la Internet se han desarrollado diversos esquemas de etiquetado de información digital en función de los recursos que han ido apareciendo.

Quien organiza información reconoce que los metadatos aseguran que los datos pueden administrarse, recuperarse, usarse, controlar el acceso y reusarse. No obstante, el rol que desempeñan en la estructura básica de la Internet hace posible llevar a cabo actividades como el comercio electrónico y la construcción de la Web semántica. Facilitan la recuperación y la interoperabilidad entre varios sistemas, y aseguran la administración de los derechos tanto de un recurso como de su contenido intelectual; en resumen, los propósitos de los metadatos ${ }^{12}$ son diversos. Por ejemplo, sirven para identificar un recurso, para asegurar la duración del contenido, para establecer la

10 Chris Oliver, Introducing RDA a guide to the basic, 2.

11 Ibid., 2-3.

12 G. G. Chowdhury y Sudatta Chowdhury, Organizing information: from the shelf to the web, 142 . 
estructura y el contexto del contenido, para auxiliar al usuario en el descubrimiento, recuperación y entrega del recurso, entre otros más.

En la administración de la información, la importancia de los metadatos radica en facilitar el registro de las propiedades descriptivas del recurso; resuelven los problemas de recuperación, otorgando a los acceso temáticos los elementos que permiten el ingreso del dato correcto; mantienen los datos por bloque, lo que en la arquitectura de la información se aprovecha para desarrollar los medios necesarios con el fin de hacer efectiva la recuperación. Por todo lo anterior, los metadatos tienen la potencialidad de transferir datos y así asegurar la interoperabilidad e intercambio de información entre sistemas.

Diremos también que los metadatos son valiosos en la arquitectura de la información porque sin ellos los problemas de organización serían comunes, debido a que al utilizarlos equivocadamente o por error se estaría frente a la imposibilidad de encontrar y recuperar correctamente la información. Chowdhury y Chowdhury ${ }^{13}$ señalan que si los metadatos no se elaboran de manera intuitiva y fácil, los usuarios difícilmente los emplearán en los programas (software), sistemas y recursos de información digital.

A propósito del acceso, se ha dicho que la arquitectura de la información debe actuar de manera decisiva en todas las organizaciones porque orientará el acceso, uso y la compartición apropiada de la información e incrementará el volumen de información digital; sin coordinación y planeación al crear y administrar la información, los esfuerzos que se realicen en desarrollar metadatos en periodos cortos provocarán caos y dificultades en la búsqueda y uso de la información. Si son empleados estándares con características locales y no aquellas consensuadas nacional e internacionalmente, se correrá el riesgo de perderse en la Internet debido a que la elaboración de vocabulario para describir y representar los recursos no estará debidamente normalizada.

Es evidente que con el surgimiento y desarrollo de la arquitectura de la información se abrió un nuevo campo de estudio y trabajo en la organización de la información, la computación, la Internet, entre otras disciplinas; se ha comprobado que esta temática tan particular emplea tanto tradiciones bibliotecarias como aquellas propias de la computación. Quien esté interesado en profundizar en el tema de los metadatos deberá, entonces, poner particular atención en cuál es el progreso de la arquitectura de la información.

En definitiva, el acercamiento al acceso a la información desde la nueva visión de los atributos del lenguaje del documento, la actualización del Principio Cardinal en la catalogación, la aparición de los lineamientos para la descripción y acceso, los metadatos como estándar para describir y represen- 
tar recursos de información digital y la administración y arquitectura de la información nos permiten estar frente a una batería de habilidades que de no aprehenderse y apropiarse idóneamente se quedará fuera del arte y la ciencia de la organización de información digital.

\section{El usuario como etiquetador de información}

Desde hace más de 150 años el almacenamiento y recuperación de la información han sido consideradas como las funciones básicas para un adecuado uso de los datos del documento. Han sido los derroteros que la organización de la información estableció como principios a seguir para acoger las necesidades que tiene el usuario al momento que emplea el catálogo de la biblioteca. En esas funciones básicas quedaron plasmadas las ideas expresadas por Cutter respecto a los objetivos del catálogo y aquéllas otras recalcadas por Svenonius ${ }^{14}$ sobre la preparación de los registros sustitutos para que los usuarios conozcan qué existe en la colección de la biblioteca. De igual forma, podemos decir que con la aparición del modelo de los Requerimientos Funcionales y el escenario de la catalogación automatizada, así como con el surgimiento de recursos de información que no necesariamente se limitan a los libros, los objetivos del nuevo catálogo tendrían que actualizar las cuatro tareas básicas -encontrar, identificar, seleccionar y obtener- que realiza el usuario para tener acceso ya no sólo al registro bibliográfico sino a la información contenida en cada recurso de información.

A consecuencia de la última actualización de los principios que gobiernan al catálogo, surgen diversas preguntas ${ }^{15}$ que, hasta la fecha, no encuentran respuestas concluyentes. Cuestionamientos que invitan a la reflexión respecto a cómo deben elaborarse los registros para los diferentes tipos de formatos y formas de los recursos de información, qué elemento descriptivo deberá registrarse como entrada en el catálogo, cuáles serán los puntos de acceso o palabras claves, si todos los elementos descriptivos son potencialmente recuperados y en qué orden tendrán que aparecer.

Por el momento, no responderemos cada uno de los cuestionamientos anteriores, puesto que el interés particular de este trabajo es adentrarse en la manera en que el usuario, no bibliotecario, está participando en el etiquetado de los recursos de información digital, puesto que hallamos planteamientos velados en el modelo de los Requerimientos Funcionales que señalan que el usuario será capaz de emplear de forma local o vinculatoria todas aquellas

14 Svenonius, op. cit., 15-30.

15 Chowdhury y Chowdhury, op. cit., 31. 
manifestaciones y representaciones de una obra, y podrá emplear distintas instancias específicas ${ }^{16}$ de una obra para su identificación. Tampoco nos detendremos en el tema de los formatos bibliográficos, como es MARC21, puesto que dicho formato nos permite crear registros estandarizados que están dirigidos por normas que permiten la creación e intercambio de los registros bibliográficos, como es del conocimiento de la comunidad bibliotecaria internacional. Concordamos con Chowdhury y Chowdhury ${ }^{17}$ en que los formatos bibliográficos tienen un rol fundamental en la creación, administración e intercambio de los registros. Lo que interesa en este trabajo es contextualizar el aprovechamiento del etiquetado de los datos de manera libre o comunitaria, conocido en el entorno de las redes sociales en línea como etiquetado social.

La variedad de recursos de información digital que se encuentra disponible tanto en las bibliotecas digitales, en los repositorios digitales como en la Internet ha propiciado la aparición de alternativas para que éstos sean descritos y representados casi de manera inmediata a su puesta en funcionamiento. Sus particularidades exigen reglas especializadas y sistemas de etiquetado.

Hemos señalado que el aprovechamiento de los metadatos radica en entender de una manera distinta cómo usar los datos de los recursos, cómo la hipertextualidad del recurso facilitará su interconectividad y cómo la mayoría de los sistemas de metadatos transcriben los datos inherentes a una entidad. La mayoría de las veces el usuario final ignora todo aquello que define un sistema de metadatos: si está correctamente definida la estructura de los elementos, si con la información que provee se logrará el uso eficiente de las entidades y si el sistema está agrupado en sus tres niveles básicos para cumplir con las tareas de encontrar, identificar, obtener y acceder al recurso de información. Quien sí debe interesarse y conocer lo anterior son los bibliotecarios profesionales, cuya tarea principal es organizar la información.

El etiquetado social ${ }^{18}$ o comunitario recomienda al usuario final que de manera superficial revise asuntos como la arquitectura de la información, el software social, la manera en que se relaciona el etiquetado con la administración de la información personal, el marcado social de los libros y los procedimientos a seguir para compartir las colecciones de objetos digitales, así como las condiciones que fija el comercio electrónico (e-commerce) para la adquisición de algún bien de consumo.

16 En el tecnicismo del modelo conceptual de los Requerimientos Funcionales, se ha denominado instancia específica (atributo) a lo que en catalogación se conoce e identifica como elementos descriptivos de las entidades. Escamilla González los denominó en su Interpretación catalográfica de los libros como los elementos más importantes para elaborar la ficha catalográfica. 
Entre los principios de las redes sociales en línea se indica que el usuario debe etiquetar su información con el fin de equilibrar el funcionamiento entre el sistema y quien interactúa. Por eso es que nos preguntamos ¿qué motiva al usuario a etiquetar sus recursos? ¿Cuáles son los debates recientes sobre el etiquetado social y aquél que realiza un profesional? ¿Cuáles son los aspectos que deben interesar al usuario cuando se enfrenta al etiquetado social?

Smith ${ }^{19}$ refiere que existen cinco motivaciones para que el etiquetador social conviva con el sistema de etiquetado. Sin explicar aún las motivaciones, refiere que la más sólida es que las etiquetas sean fáciles de usar y se invierta un mínimo de tiempo:

- Etiqueta simple. Con base en esta idea es que el etiquetador social puede crear múltiples rutas de acceso para recuperar sus recursos y así agregar más de una etiqueta.

- Etiqueta flexible. Que puedan adaptarse a cualquier situación, propósito y clase de información.

- Etiqueta extendible. Que en ningún momento se niegue o prohíba la creación de nuevas etiquetas y que al querer describir algo nuevo se pueda hacer sin impedimentos.

- Etiquetas que puedan agregarse. Los diferentes tipos de folders proveen información local, pero con las etiquetas que permiten agregar información de otros sitios podrán emplearse para reunir información en torno a múltiples sitios web.

- Recomendación. No todos los que ingresan al sistema de etiquetado conocen el significado de cada etiqueta, por lo que es necesario agregar breves explicaciones sobre el uso.

Respecto a los asuntos que deben interesar al etiquetador cuando ingresa al uso de los metadatos sociales, coincidimos con Smith ${ }^{20}$ cuando refiere que la arquitectura de la información, el software social y la administración de la información personal son los ejes de una buena práctica.

Sobre la arquitectura de la información, el etiquetado deberá fijarse porque es donde reside el diseño estructural para compartir la información y permite enfocarse en el vocabulario controlado, los sistemas de búsqueda y descubrimiento, así como la consistencia de los esquemas de navegación. 
Respecto al software social, se le pide al etiquetador que identifique las variantes existentes en cada software; unos son más sencillos que otros pero su intención es que los usuarios convivan con el sistema de una manera fácil e interactiva.

Finalmente, el etiquetador ha de ver la administración de la información personal como una manera por medio de la cual pueda adquirir, mantener, recuperar y usar la información de las instancias específicas de cada documento.

En cuanto a las tensiones que enfrenta esta visión del etiquetado social y las prácticas que siguen la catalogación y otras subdisciplinas similares, percibimos su ubicación principalmente en cuatro puntos: entre lo personal y lo social, entre lo idiosincrático y lo estándar, entre lo libre y lo controlado, y entre lo amateur y lo experto. Smith concluye que desde la perspectiva que se mire siempre se encontrarán puntos de tensión porque los sistemas son creados con distintos propósitos, valores económicos y perspectivas del etiquetado. De modo tal que el etiquetado en el modelo social o colaborativo estará imperando en sistemas, repositorios o sitios web que operen con estructuras sencillas y de fácil comprensión.

\section{El prosumidor de información, ¿quién es y qué hace por el acceso a la información?}

El primer camino lógico establecido para crear un sistema de metadatos ${ }^{21}$ es analizar el contenido, determinar el usuario y decidir respecto a los Requerimientos Funcionales. Esto mismo se observa en el etiquetado social, ${ }^{22}$ el cual requiere de tres elementos principales para conformar una red social en línea: el usuario, los recursos y el etiquetado.

Ambos fundamentos refieren que es valioso identificar anticipadamente las necesidades y recursos de información tanto del grupo de usuarios principales como secundarios. Reconocidos cada uno de ellos, será posible la determinación de los atributos de los recursos digitales que emplean para encontrar e identificar sus documentos.

En el caso del desarrollo de colecciones digitales ${ }^{23}$ la forma en que se lleva a cabo el trabajo con usuarios es por medio de un diseñador de metadatos, quien trabaja de manera conjunta con un experto o curador en el tema. Ellos decidirán los usuarios de una colección en particular, las necesidades para navegar y buscar por ubicación geográfica y tiempo, navegar por un conjunto se-

21 Steven J. Miller, Metadata for digital collections, 253.

22 Smith, op. cit., 39-51.

23 Miller, op. cit., 254. 
lecto de categorías temáticas relevantes en una colección en particular, limitar las búsquedas por un rango de fecha y por el tipo de recursos primarios.

En las redes sociales en línea ${ }^{24}$ pueden considerarse tres tipos de conexiones entre los usuarios, a saber:

- Seguidores. Son aquellos que simplemente hacen contacto entre los usuarios.

- Contactos. Aquellos que tiene comunicación recíproca con los usuarios de la red.

- Grupos. Aquellos que comparten conjuntamente los recursos sobre un tema en particular. A su vez, pueden formarse subgrupos a solicitud de algún miembro del grupo.

Los contextos anteriores sirven de antesala para referirnos a las características principales que asumirá un usuario al momento de transitar hacia prosumidor, esto en la consonacia entre el sistema de etiquetado y quien marcará algún recurso de información.

Los modelos teóricos de la década de los ochenta e inicios de los noventa no podían reconocerse aún como los más idóneos para ubicar al prosumidor debido a que la Internet no se popularizaba como una nueva forma de comunicación masiva. Fue hasta inicios del 2000 que las condiciones favorecerían la consolidación del prosumidor. La Internet había introducido cambios significativos al modelo tradicional de producción y distribución de información; había modificado el modelo de tal forma que la manera común basada en la distribución de bienes y servicios físicos se veía alterada por la obtención de bienes que carecían de una base física.

A diferencia del modelo tradicional, el emergente permitía el acceso a los recursos de información ${ }^{25}$ con una retroalimentación desigual. Es decir:

- La relación entre productores y consumidores estaba desproporcionada debido al dominio en los canales de distribución de los productores.

- El acceso a los medios de producción y distribución de la información estaba disponible, aunque limitado a un pequeño grupo de operadores, lo que no favorecía las prácticas comerciales.

- El mismo impulso tecnológico estaba propiciando que las comunicaciones persona a persona fueran cambiado debido a la incorporación

24 Smith, op. cit., 44-45.

25 Axel Burns, Blogs, Wikipedia, Second Life, and Beyond: from Production to Produsage, 13. 
de modelos peer-to-peer, los cuales empezarían a enriquecer la comunicación colaborativa, la producción y distribución a escala global.

- En el formato digital, los contenidos son más fáciles y rápidos de compartir y de modificar, por este motivo el concepto de "consumo" en su sentido tradicional no aplicaba, de ahí que la información digital no tuviera rival a vencer.

Si el modelo de producción y distribución de la información ha cambiado debido al vertiginoso desarrollo de la información digital, ésta requiere de un método donde el usuario sea el principal artífice de su creación y actualización. La pregunta por resolver es, entonces, ¿cuáles son las características del nuevo usuario, identificado como prosumidor?

Burns $^{26}$ explica que antes de llegar a ser un prosumidor debe pasarse obligatoriamente por un estado híbrido para relacionarse con la información digital. En términos tradicionales solamente son usuarios productores pero que no llegan al extremo de ser productores y consumidores al mismo tiempo.

De acuerdo con Burns ${ }^{27}$ los cuatros principios que identifican a un prosumidor son:

- Participación abierta. Evaluación de la comunidad. Muchos de los participantes son capaces de examinar, evaluar y agregar contribuciones a los comentarios que le precedieron, lo que trae como resultado un mejor resultado e incrementa la calidad de la discusión.

- Fluid Heterarchy, meritocracia Ad Hoc. Un prosumidor necesariamente procede del principio descrito como equipotencialidad, el cual señala que se cuentan con las habilidades y capacidades pues no todos los prosumidores participantes son iguales, pero tienen una habilidad igual para hacer una contribución digna al proyecto.

- Hechos sin concluir, procesos continuos. Debido a que se adopta un modelo probabilístico los participantes se ven envueltos en un trabajo equipotencial. Se observa que los proyectos son creados de forma gradual, las tareas modulares invitan a los prosumidores a contribuir de manera casual en la producción de contenidos colaborativos, que se transmiten y comparten en espacios de información con acceso abierto, de ahí que el proceso del prosumidor tienda a ser continuable infinitamente. 
- Propiedad común, recompensas individuales. Como se ha señalado, en una comunidad de prosumidores la información debe ser necesariamente compartida y valorada por quienes crean los contenidos con miras a que el proceso de creación deba permanecer disponible para todos los futuros participantes, quienes estarán preparados para incorporar sus contribuciones. Claro está que, si se desea participar como prosumidor híbrido, deberán acatarse las disposiciones morales y legales que se incluyen en el GNU (General Publica License and Free Documentation License), el Open Source License y el Creative Commons License. Tales documentos estipulan, por ejemplo, que el contenido comunitario deberá estar libremente disponible, que las modificaciones de dichos contenidos tendrán que realizarse bajo las mismas condiciones y que las contribuciones de los prosumidores individuales deben ser reconocidas y (cuando sea pertinente) recompensadas.

Desde hace un par de lustros, el prosumidor de información ha comenzado a aparecer en las instituciones, organizaciones y empresas. Se está generando un proceso de convergencia, inverosímil para todos, a tal grado que está naciendo la idea de la Convergencia Cultural ${ }^{28}$ (Cultural Converge) en donde la cultura actual pierde su posición, privilegiando a la parte final de la cadena de producción con el propósito de reducir los niveles de participación de todos aquellos que se encuentran en la Red.

Conviene señalar que el modelo de Convergencia Cultural está prosperando en las empresas de reciente creación, las cuales tiene como visión de trabajo instituir entornos denominados "ganar-ganar" para que los consumidores y las mismas empresas lo perciban como un aspecto clave que da popularidad. Se promueven, por ejemplo, servicios sofisticados que requieren del apoyo de especialistas como los diseñadores de sistemas de información en red.

Dicho de otro modo, Jackson ${ }^{29}$ refiere que la presencia de la Web 2.0 en las empresas facilita aprovechar las aplicaciones desarrolladas por esta tecnología:

- Con un uso responsable en la creciente información y proliferación de correos electrónicos, puesto que esto puede generar grandes retornos de conocimiento como producto valioso a la empresa.

- Responder a las expectativas e inclinaciones de las siguientes generaciones de trabajadores.

28 Idem.

29 Paul Jackson, Web 2.0 knowledge technologies and enterprise: smarter, lighter and cheaper, $92-$ 93. 
- Asumir la pérdida de conocimiento cuando comiencen a retirarse los miembros de la generación denominada baby boom.

- Entender las oportunidades que ofrece la conectividad y las redes dinámicas.

- Contar con la capacidad y el conocimiento para crear productos sofisticados que permitan algunas pequeñas contribuciones.

- Razonar sobre la fragmentación de la cadena de valor del trabajo y la creación de multitareas.

- Entender la proliferación del trabajo outsourcing para ciertos contratos y movilidad de los miembros del equipo de trabajo.

De lo anterior debe entenderse que mucho depende del sistema de información con que se cuente, porque de una buena decisión al adquirir e implementar las soluciones tecnológicas dependerá el funcionamiento de las aplicaciones de la Web 2.0.

A causa de las características del prosumidor de información y la convergencia cultural, es de suponer que el discurso digital ha dado un vuelco, a tal grado que su estructura toma una especial relevancia. En nociones amplias, lo que se ha llegado a subrayar es el incremento en el uso de acrónimos y emoticones como medio de expresión. También se ha detectado que los discursos digitales están más bien dentro de los límites de la oralidad y la literatura tradicional. De igual forma, el uso prefigurativo de gesticulaciones está empleándose como estilo transicional, colocándose como parte de las normas de participación. Cercanas a estas particularidades del discurso digital se encuentran las folsonomías (folksonomy) o las wiki, las cuales pretenden aportar ideas acerca del orden dentro del caos que se está generando a partir de la estructura del discurso digital.

En definitiva, el prosumidor de información no debe perderse de vista en la generación de contenidos digitales; debe observarse como un usuario que demanda más información en comparación con el usuario pasivo e híbrido; debe asumirse que su conocimiento y uso de las tecnologías de información es mayor, escenario que abre posibilidades para presuponer que sus valores culturales, prácticas, tradiciones y creencias están soportadas en la cultura de la información digital.

\section{LA COMPOSICIÓN DE LAS PAUTAS PARA ACCESO A LA INFORMACIÓN}

Muchas de las discusiones vertidas en este trabajo giran en torno a tres ideas: el acceso a la información, los sistemas de etiquetado y la resignificación del 
lenguaje del documento, y el prosumidor de información como artífice de la cultura y el discurso digital.

El acceso a la información ha cambiado, hay nuevos modelos de producción, distribución y consumo debido a que la información digital presenta características distintas a las tradicionales. Pero este modelo no apareció sino hasta que los expertos lo acreditaron y reconocieron como una nueva práctica para producir y distribuir información. Además, ha sido necesario que los conocimientos sobre las actividades económicas basadas en el acceso, la convergencia cultural y la composición de la información digital fueran apropiados por las diversas comunidades con el fin de establecer una nueva categoría para la alfabetización digital y las capacidades en torno de las cuales el nuevo usuario desarrollará sus habilidades respecto de las prácticas que sigue un prosumidor.

En el momento en que nos encontramos, el acceso a la información mediante el nuevo modelo de producción, como los blogs, wikis o podcasts, ha evolucionado los formatos existentes de información. Por igual se encuentran las redes sociales como Facebook y los motores de búsqueda como Google, los cuales carecen de algún punto de comparación en el mundo de la información análoga.

Browing ${ }^{30}$, Braman $^{31}$ y Block ${ }^{32}$ señalan que la transición digital ha provocado que bibliotecarios, editores y libreros reexaminen sus roles. Algunas bibliotecas observaron cómo las colecciones comenzaban a "salir" del copyright al ser distribuidas de manera digital. Esto llevó a que varias librerías y casas editoriales ingresaran al esquema de free-for-copy o pay-for-play. Este cambio, con el paso del tiempo, ha demostrado que para la biblioteca no es fácil establecerse en el contexto digital, pero para los libreros y editores es un poco más sencillo, siempre y cuando se resuelva el asunto de las compensaciones en la propiedad intelectual.

En síntesis, el acceso a la información examinado desde la perspectiva de la economía en red y los nuevos modelos de producción ha puesto en la discusión la aparición de la convergencia cultural y el surgimiento del prosumidor de información.

En la organización de la información se identificó que las nuevas pautas para el acceso se están fijando desde la perspectiva de los sistemas de etiquetado, los metadatos y el usuario como etiquetador de la información digital. La percepción tradicional sobre la organización de la información es maxi-

30 John Browing, "What is the role of libraries in the information economy?", 55.

31 Sandra Braman. "Theorizing the impact of it on libraries-state relations", 105.

32 Maryline Block (ed.), Net effects: how librarians can manage the unintended consequences of the Internet. 
mizar la utilidad social de los registros bibliográficos para beneficio del usuario, aun teniendo que recurrir al uso de técnicas y procedimientos que, a causa del crecimiento exponencial de la información, las condiciones de vida y el uso de las tecnologías de la información, se encuentran al borde del desuso.

Pese a lo anterior, en nuestros días es común hablar sobre la World Wide Web, Internet, biblioteca digital, repositorios digitales, XML, Web 2.0, tecnología móvil, web semántica, entre otros asuntos más. Técnicamente éstos expresan cómo las tecnologías de la información protagonizan el escenario con relación al acceso a la información en la biblioteca.

Sin haber explicado los aspectos técnicos y el funcionamiento de cada uno, nos percatamos que hay tensiones entre los sistemas de etiquetado públicos y privados. Las discrepancias entre el etiquetado amateur y el experto continuarán en tanto que no sigan un mismo método, al igual que entre los sistemas de etiquetado idiosincráticos y normados, que no siguen los mismos principios.

En el contexto de la tecnología web y las bibliotecas digitales, Chowdhury y Chowdhury ${ }^{33}$ aclaran que las ontologías están jugando un papel significativo debido a que sus mecanismos permiten el análisis del significado de los recursos favoreciendo su desempeño en el proceso de acceso a la información, así como al organizar y reunir aquella información heterogénea contenida en los recursos de información digital. El propósito de estas iniciativas es facilitar la organización y procesamiento de la información digital, otorgándole significado para permitir su uso, acceso y recuperación en el desarrollo de la web semántica. Igualmente, señalan que la facilidad de contar con esquemas de metadatos permite a los usuarios etiquetar su información (tagging) como lo hemos puntualizado con los prosumidores, dando origen a sistemas de clasificación social o folsonomías. En estos sistemas, el usuario puede etiquetar y clasificar los recursos.

Un elemento clave es que las tecnologías web se basan en lenguajes de marcado conocidos como XML. Éste sirve de fundamento para llevar a cabo la creación de los sistemas de información basados en la Web. De acuerdo con Clarke, ${ }^{34}$ XML tiene aplicaciones en diversos niveles que van desde el análisis de los documento como materia prima hasta las sofisticadas interfaces que interactúan en la Web.

Si estamos pensando en la biblioteca del futuro con prosumidores de información y nuevos modelos para el acceso, entonces todos los esfuerzos actuales deben orientarse para entender lo que ofrece XML a los servicios que 
proporciona la biblioteca. Así, según Miller y Clarke, ${ }^{35}$ conviene revisar los XSLT o CSS, pues cada uno de ellos constituye un apoyo capaz de reconfigurar el medio en el cual trabaja la información digital. En síntesis, la creación de manuales de referencia de XML ayudará a resolver y reconsiderar los medios que hay entre el contenido y la presentación de los recursos y entre los productos y servicios, como bien se señaló en las características del usuario como etiquetador.

El prosumidor emerge de los entornos en línea y en red, donde participa no solamente de manera pasiva como consumidor, sino como un usuario activo que tiende a participar con un interés fuertemente personal; en algunos casos participa activamente más enfocado en la productividad de las redes sociales y contenido comunitario, de ahí su importancia en el etiquetado social.

\section{BiBLIOGRAFÍA}

Block, Maryline, ed., Net effects: how librarians can manage the unintended consequences of the Internet. Medford, New Jersey: Information Today, 2003.

Braman, Sandra. "Theorizing the impact of it on libraries-state relations”, en Gloria J. Leckie y John E. Buschman, eds., Information Technology in Librarianship: New Critical Approach. Westport, Conn.: Libraries Unlimited, 2009.

Browing, John. "What is the role of libraries in the information economy?", en Mark Stefik. Internet dreams: archetypes, myths and metaphors. Chicago: The MIT Press, 1997.

Burns, Axel. Blogs, Wikipedias, Second Life, and Beyond: from Production to Produsage. New York: Peter Lang, 2008.

Chan, Lois Mai y Edward T. O’Neil. FAST: faceted application of subject terminology: principles and applications. Santa Barbara, California: Libraries Unlimited, 2010.

Chowdhury, G. G. y Sudatta Chowdhury. Organization information: from the shelf to the web. London: Facet Publishing, 2007.

Clarke, Kevin S. "Updating MARC records with XMLMARC", en Roy Tennant, XML in libraries. New York: Neal-Schuman Publishers, 2007, 3-13.

Curran, Kevin, ed., Understanding the Internet: a glimpse into the building blocks, applications, security and hidden secrets of the Web. Oxford: Chandos Publishing, 2009. 
Escamilla González, Gloria. Interpretación catalográfica de los libros. México: UNAM, 1987.

Jackson, Paul. Web 2.0 knowledge technologies and enterprise: smarter, lighter and cheaper. Oxford: Chandos Publishing, 2010.

Kuhn, Thomas. La estructura de las revoluciones cientificas. México: Fondo de Cultura Económica, 2007.

Leckie, Gloria J. y John E. Buschman. Information technology in librarianship: new critical approaches. Westport, Connecticut, 2009.

Miller, Dick R. y Kevin S. Clarke. Putting XML to work in the library: tools for improving access and management. Chicago: American Library Association, 2004.

Miller, Steven J. Metadata for digital collections: a how-to-do-it- manual. New York: Neal-Schuman Publisher, 2011.

Oliver, Chris. Introducing RDA: a guide to the basics. United States of America: Facet Publishing, 2010.

Perales Ojeda, Alicia. De la informática. México: Universidad Nacional Autónoma de México, 1975.

Rifkin, Jeremy, La era del acceso: la revolución de la nueva economía. Barcelona: Paidós, 2002.

Rodríguez García, Ariel Alejandro. Las nuevas entidades de información analizadas desde la perspectiva de la organización de la información. México: UNAM, Centro de Universitario de Investigaciones Bibliotecológicas, 2010.

Schamber, Linda. "What is a document? Rethinking the concept in uneasy time", JASIS 47 (9) (1996): 669-671.

Segev, Elad. Google and the digital divide: the bias of online knowledge. Oxford: Chandos Publishing, 2012.

Stefik, Mark. Internet dreams: archetypes, myths, and metaphors. Cambridge, Massachusetts, 1996.

Smith, Gene. Tegging: people-powered metadata for the social web. Berckley, Ca.: New Riders, 2008.

Svenonius, Elaine. The intellectual foundation of information organization. Cambridge, Mass.: The MIT Press, 2000.

Tennant, Roy, ed., XML in libraries, New York: Neal-Schuman Publishers, 2002.

Torres Vargas, Georgina Araceli. El acceso universal a la información, del modelo librario al digital. México: Universidad Nacional Autónoma de México, 2010.

Tredinnick, Luke. Digital information cultura: the individual and society in the digital age. Oxford: Chandos Publishing, 2008.

Vargas Llosa, Mario. La civilización del espectáculo. México: Alfaguara, 2012. 
Para citar este artículo:

Rodríguez García, Ariel Alejandro. 2016. "Las nuevas pautas para el acceso a la información." Investigación Bibliotecológica: Archivonomía, Bibliotecología e Información 69: 121-141. http://dx.doi.org /10.1016/j.ibbai.2016.04.015 
